Research Paper

\title{
ERRa Regulates OTUB1 Expression to Promote Colorectal Cancer Cell Migration
}

\author{
Yi Zhou' ${ }^{1}$ Qingzhu Jia ${ }^{1}$, Xiaoqing Meng², Diangang Chen ${ }^{1}$, Bo Zhu1 ${ }^{\bowtie}$ \\ 1. Institute of Cancer, Xinqiao Hospital, Third Military Medical University, Chongqing, China \\ 2. Department of Hematology, the Third Affiliated Hospital of Chongqing Medical University (Gener Hospital), Chongqing, China \\ $\triangle$ Corresponding author: Bo Zhu, Institute of Cancer, Xinqiao Hospital, Third Military Medical University, Chongqing, China, Postcode: 400037. Tel: \\ +8613594611534; Email: bo.zhu@tmmu.edu.cn \\ (C) The author(s). This is an open access article distributed under the terms of the Creative Commons Attribution License (https://creativecommons.org/licenses/by/4.0/). \\ See http:/ /ivyspring.com/terms for full terms and conditions.
}

Received: 2019.06.08; Accepted: 2019.08.20; Published: 2019.10.06

\begin{abstract}
Ovarian tumor domain-containing ubiquitin aldehyde binding protein 1 (OTUBI) is overexpressed in many cancers and plays an important role in tumor progression and metastasis. However, the molecular mechanisms underlying OTUB1 overexpression are not clear. In this study, we found that estrogen-related receptor alpha (ERRa, also called NR3BI) binds to OTUBI promoter and regulates its expression in colorectal cancer. Furthermore, ERR $\alpha$ promoted the migration of CRC cells by inducing vimentin expression via OTUB1. Our data show that OTUB1 is a novel target of ERRa and indicate that ERRQ-OTUBI signaling may play a significant role in CRC metastasis.
\end{abstract}

Key words: OTUB1, ERRa, colorectal cancer, transcriptional regulation, cell migration

\section{Introduction}

Colorectal cancer $(\mathrm{CRC})$ is the fifth most common cancer in China: in 2015, the incidence of CRC in China was about 376,300 new cases, and the mortality was 191,000 cases [1]. The primary factor causing death in patients with CRC is metastasis. Therefore, understanding the metastatic mechanisms in CRC is important for improving treatment strategies.

Estrogen-related receptor alpha (ERRa, also called NR3B1), encoded by the ESRRA gene, is a member of the ligand-independent orphan nuclear receptor superfamily [2]. ERRa shares high sequence identity with ERa, but a genome-wide binding site location analysis indicated that most of the genes regulated by ERRa are distinct from those regulated by ERa [3]. ERRa regulates the expression of genes related to cell metabolism, such as those involved in the tricarboxylic acid cycle, aerobic glycolysis, and lipogenesis [4-7]. However, recent studies have shown that ERRa might not only regulate metabolism, but might also participate in the proliferation and metastasis of cancer cells. For example, ERRa promotes cellular migration by activating the expression of TNFAIP1 and subsequently destabilizing RHOA [8]. The activity of ERRa is associated with the WNT signal. In this regard, a study has shown that transcriptional upregulation of WNT11 by ERRa influences the migration of cancer cells [9]. ERRa plays an important role in the development of hormone-dependent cancers. ERRa levels are higher in breast, ovarian, cervical and prostate cancer tissues than in normal tissues, and patients with high ERRa expression have poor survival [10-13]. Moreover, ERRa promotes cancer proliferation and metastasis in non-hormonedependent tumors, such as oral squamous cell carcinoma [14]. In CRC, the mRNA levels of ERRa are higher in tumor tissues than in normal mucosa, and increase significantly from TNM stage II to IV [15]. Furthermore, high ERRa expression is significantly associated with an increased risk of recurrence and poor prognosis in CRC [16].

Ovarian tumor domain-containing ubiquitin aldehyde binding protein 1 (OTUB1) is a deubiquitinating enzyme that belongs to the OTU family of cysteine proteases. By specifically regulating 
Lys48-linked ubiquitin and Lys63-linked polyubiquitin, OTUB1 can target and inhibit E2 enzymes, such as UBC13, UBE2D, and UBE2E. OTUB1 regulates naive CD4+ T cell proliferation via GRAIL [17]. OTUB1 is also a regulator of p53: by suppressing MDM2mediated p53 ubiquitination, it stabilizes and activates p53, induces cell apoptosis and inhibits cell proliferation [18]. Additionally, by stabilizing MDMX expression, OTUB1 enhances p53 phosphorylation at S46 and promotes the mitochondria-mediated apoptotic pathway [19]. Recent studies have shown that OTUB1 plays a crucial role in tumorigenesis and cancer progression. By deubiquitinating and stabilizing phospho-SMAD2/3, induced by TGF $\beta$, OTUB1 efficiently regulates TGF $\beta$-mediated cell migration [20]. By cleaving K48-linked polyubiquitin chains, OTUB1 regulates c-IAP1 expression and TWEAK-induced activation of canonical NF- $\mathrm{KB}$ and MAPK signaling pathways and modulates TNFdependent cell death [21]. We have previously shown that OTUB1 is overexpressed in CRC and high OTUB1 expression correlates with short survival [22]. In this study, we evaluated the association between ERRa and OTUB1 expression in CRC and elucidated the mechanism through which ERRa regulated OTUB1 expression to promote CRC migration.

\section{Materials and Methods}

\section{Cell lines and culture conditions}

The human colon cancer cell lines HCT116 and Caco2 were obtained from the American Type Culture Collection. HCT116 cells were cultured in RPMI 1640 supplemented with $10 \%$ fetal bovine serum (FBS), and Caco2 cells were cultured in Dulbecco's modified Eagle's medium (DMEM) supplemented with 10\% FBS. All cells were maintained in a humidified 5\% $\mathrm{CO}_{2}$ atmosphere at $37^{\circ} \mathrm{C}$.

\section{Cell transfection}

The pcDNA3.1-OTUB1 plasmid was generated amplifying OTUB1 from HCT116 using the primers 5'-ATTGGATCCACCATGGCGGCGGAGGAACCT$3^{\prime}$ and $5^{\prime}$-ATGCTCGAGCTATTTGTAGAGGATATCG TA-3'. The amplified DNA was digested with BamHI and XhoI and inserted into the pcDNA3.1 plasmid.

The pcDNA3.1-ERRa plasmid was generated for amplifying ERRa from HCT116 using the primers 5'-TAGGATCCACCATGTCCAGCCAGGTGGTG-3' and 5'-GCGAATTCTCAGTCCATCATGGCCTC-3'. The amplified DNA was digested with BamHI and EcoRI and the released fragment was inserted into the pcDNA3.1 plasmid.

The OTUB1 siRNA sequences were \#1 (5'-UUAA CTGUCUGGCCUAUGATT-3') and \#2 (5'-CCAUGU
GCAAGGAGAGCGATT-3') and the negative control (NC) siRNA was 5'-UUCUCCGAACGUGUCACGU TT- $3^{\prime}$. The ERRa siRNA sequences were \#1 (5'-UGGU GGGCAUUGAGCCUCUCUACAU-3') and \#2 (5'-GA AUGCACUGGUGUCUCAUCUGCUG-3') and 5'-GG UAGGUGAGUGUACAGACGCAAUA-3' was the NC siRNA [9].

For transient transfections, $6 \times 10^{5}$ HCT116 or $4 \times$ $10^{5}$ Caco2 cells were seeded in 6-well plates. Twenty-four hours later, the cells were transfected with $4 \mu \mathrm{g}$ of plasmid DNA or $100 \mathrm{nM}$ siRNA using Lipofectamine $^{\mathrm{TM}} 2000$ (Invitrogen) according to the manufacturer's protocol. After $48 \mathrm{~h}$, the cells were collected for quantitative PCR (qPCR), western blotting, fluorescence, Chromatin Immunoprecipitation (CHIP) and migration assays.

\section{Western blot analysis}

Total cellular proteins were extracted in lysis buffer (1\% Triton X-100, $0.1 \%$ sodium dodecyl sulfate [pH 7.3], $50 \mathrm{mM}$ Tris, and $150 \mathrm{mM} \mathrm{NaCl}$ ) with protease inhibitors (Roche) for $30 \mathrm{~min}$ on ice and centrifuged at $16,000 \mathrm{~g}$ at $4{ }^{\circ} \mathrm{C}$. Western blots were carried out as previously described [23]. The primary antibodies used included anti-OTUB1, anti-ERRa, anti- $\beta$-actin, anti-vimentin, anti-zo- 1 , anti-snail, anti-E-cadherin and anti- $\beta$-catenin antibodies. AntiOTUB1 antibody was purchased from abcom and the other antibodies were purchased from Cell Signaling Technology and used 1:1,000.

\section{Migration assays}

To investigate the effect of ERRa and OTUB1 on the migration of colorectal cells, $6 \times 10^{5}$ HCT116 or $4 \times$ $10^{5} \mathrm{Caco} 2$ cells were seeded in 6 -well plates. Twentyfour hours later, the cells were transfected with $4 \mu \mathrm{g}$ of pCDNA3.1-ERRa plasmid DNA and 100 nM OTUB1 siRNA or $100 \mathrm{nM}$ ERRa siRNA and $4 \mu \mathrm{g}$ pCDNA3.1OTUB1 plasmid. After $24 \mathrm{~h}, 0.5 \times 10^{5}$ HCT116 cells or $1.5 \times 10^{5} \mathrm{Caco} 2$ in serum-free medium were seeded into a Boyden chamber (8- $\mu \mathrm{m}$ pore; BD Falcon) to assay cell migration. The chambers were inserted in 24-well plates with medium with $10 \%$ FBS and incubated for 24 to $36 \mathrm{~h}$ at $37{ }^{\circ} \mathrm{C}$ with $5 \% \mathrm{CO}_{2}$. The cells on the underside of filter membrane were fixed in ethanol, stained with crystal violet and counted under a microscope (Leica Biosystems ADMI300B).

\section{qPCR analysis of OTUBI expression}

Total RNA was extracted from CRC cell lines using RNAiso Plus (Takara). Two micrograms of total RNA were used to synthesize complimentary DNA (cDNA) with the PrimeScript ${ }^{\mathrm{TM}} \mathrm{RT}$ reagent Kit with gDNA Eraser (Takara), following the manufacturer's protocol. For qPCR, cDNA products were amplified 
using a SYBR Green PCR Kit (Takara). Quantification was performed using the 7500 real time PCR system (Applied Biosystems). OTUB1 expression values were normalized to $\beta$-actin and calculated using the comparative CT method $\left(2^{-\Delta \Delta \mathrm{CT}}\right)$. The primer sequences for OTUB1 were $5^{\prime}$-TTTCTATCGGGCTTT CGGA-3' (forward) and 5'-TCGGAGGTGCTCTGGTC AT-3' (reverse). The primer sequences for $\beta$-actin were 5'-TGGCACCCAGCACAATGAA-3' (forward) and 5'-CTAAGTCATAGTCCGCCTAGAAGCA-3'

(reverse).

\section{Luciferase assays}

The $2 \mathrm{~kb}-1,000 /+1,000$ OTUB1 promoter sequence was amplified by PCR using HCT116 genomic DNA with the primer pair 5'-TATACGCGT AAACCAGAAGGACACAGA-3' and 5'- TCCAGATC TAGGCGTGGTCTCTTGCAG-3', digested with MluI and BgI II, and cloned into the MluI/Bgl II site of the pGL3-basic vector (Promega) to generate the pGL3-OTUB1 plasmid. The constructs containing the mutated OTUB1 promoter sequence were generated by PCR using the $-1,000 /+1,000$ OTUB1 promoter sequence DNA as template and the primers listed in Table S1.

HCT116 or Caco 2 cells $\left(4 \times 10^{4}\right)$ were seeded in 24-well plates. Twenty-four hours later, the cells transfected with $200 \mathrm{ng}$ pGL3-OTUB1 promoter or pGL3 Basic, 10 ng pGL4.73, 200 ng pCDNA3.1-ERRa or pCDNA3.1, $10 \mathrm{nM}$ ERRa siRNA or siNC, or $10 \mu \mathrm{M}$ XCT790 or DMSO. After $48 \mathrm{~h}$, the cells were washed by PBS and solubilized with Passive Lysis Buffer (Promega), then assayed for both Firefly and Renilla luciferase activities using a Dual-Luciferase Reporter Assay System (Promega) according to the manufacturer's instructions.

\section{ChIP}

ChIP assays were performed using the SimpleCHIP ${ }^{\circledR}$ enzymatic chromatin immunoprecipitation kit (Cell Signaling Technology, \#9002) according to the manufacturer's protocol. Briefly, $12 \times$ $10^{6}$ cells were cross-linked with $1 \%$ formaldehyde for $10 \mathrm{~min}$ at room temperature. Nuclei were isolated upon cells lysis and chromatin was digested into fragments of 150-900 bp by micrococcal nuclease. The nuclear membrane was disrupted by sonication. For immunoprecipitation, 5-10 $\mu \mathrm{g}$ of total chromatin were incubated overnight at $4{ }^{\circ} \mathrm{C}$ with $10 \mu \mathrm{g}$ anti-ERRa antibodies (Cell Signaling Technology,\#13826) or normal Rabbit IgG as negative control (Cell Signaling Technology, \#2729). The antibody-DNA complexes were incubated with $30 \mu \mathrm{L}$ of ChIP-grade protein G-agarose beads for $2 \mathrm{~h}$ at $4{ }^{\circ} \mathrm{C}$, eluted, digested with Proteinase $\mathrm{K}$ for $2 \mathrm{~h}$ at $65^{\circ} \mathrm{C}$, and finally purified with spin columns. ERRa binding site on OTUB1 promoter was assessed by qPCR using the primers $5^{\prime}$-CTCTAA GAAAGGCGAGGGT-3' (forward) and 5'-CTCTTGC GGTCGTGGATA-3' (reverse).

\section{Statistical analysis}

Data were analyzed using the GraphPad Prism software V5.0 (Graphpad, San Diego, CA, USA). The Pearson's test was used for the correlation analysis. Statistical significance was determined using the two-tailed t-test, and P-values $<0.05$ were considered significant.

\section{Results}

\section{ERR $\alpha$ regulates OTUB 1 expression}

To explore the mechanisms underlying OTUB1 overexpression in CRC, we searched for transcription factors that may bind to the OTUB1 promoter (and thereby regulate OTUB1 expression) in the JASPAR database (http://jaspar.genereg.net). The correlation between OTUB1 expression and these transcription factors was analyzed using the TCGA database. We found that the expression of ERRa significantly correlated with that of OTUB1 in CRC (Figure S1).

To investigate whether OTUB1 expression is regulated by ERRa in CRC cells, we overexpressed or silenced ERRa or used the ERRa inhibitor XCT790. After transfecting HCT116 or Caco2 cells with an ERRa expression plasmid for $48 \mathrm{~h}$, ERRa was found to be overexpressed (Figure 1B). Meanwhile, the levels of OTUB1 mRNA (Figure 1A) and protein (Figure 1B) were also increased. Two siRNAs were used to knockdown ERRa expression (Figure S2). We silenced or inhibited ERRa in HCT116 or Caco2, and western blot analysis showed that, in these cells, ERRa was downregulated (Figure 1D and F) and the levels of OTUB1 mRNA (Figure 1C and E) and protein (Figure $1 \mathrm{D}$ and $\mathrm{F}$ ) were decreased. These results showed that overexpression or knockdown of ERRa significantly enhanced or suppressed OTUB1 expression.

\section{ERR a regulates OTUBI promoter activity}

To study whether the transcription of OTUB1 was regulated by ERRa, we constructed the OTUB1 luciferase reporter by inserting the $-1,000$ to $+1,000$ region of the OTUB1 gene upstream of the firefly luciferase coding sequence in the vector pGL3-Basic (Figure 2A). Luciferase activity assays showed that the OTUB1 promoter was activated by ERRa overexpression in HCT116 and Caco-2 cells (Figure 2B and E). By contrast, knockdown of ERRa expression or inhibition of ERRa through XCT790 suppressed the promoter activity (Figure 2C, D, F and G). 
A

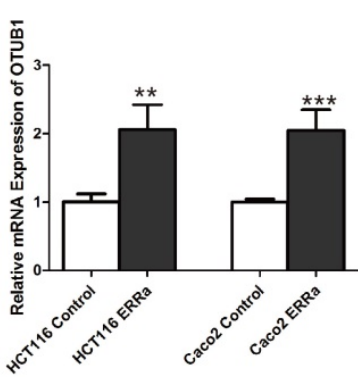

D

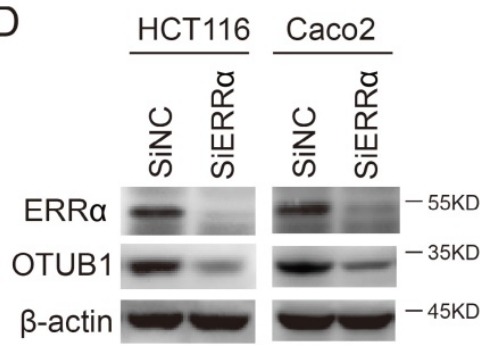

B

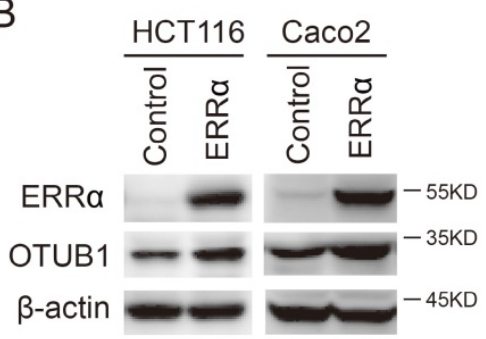

E

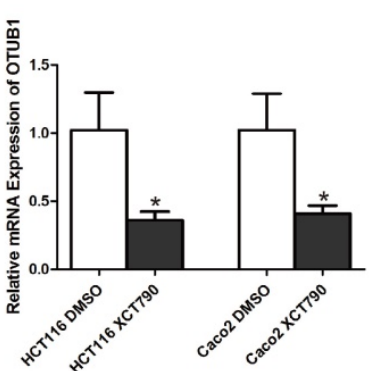

C

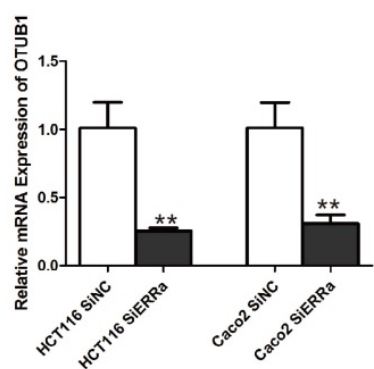

F

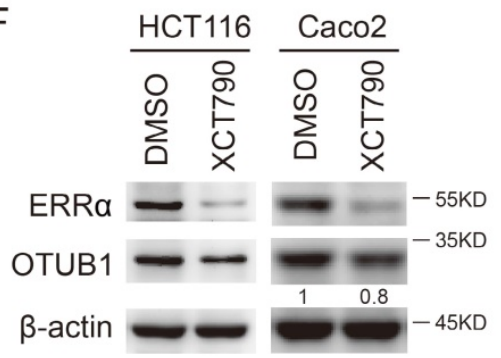

Figure 1. ERR $\alpha$ regulates OTUB 1. HCT116 or Caco2 were transfected with an empty vector or a plasmid overexpressing ERRa, treated with DMSO or XCT790 (10 $\mu M$ ), or transfected with siNC or ERRa siRNA for 48 h. OTUBI mRNA levels in HCT116 and Caco2 were measured by q-PCR (A, C, E); ERRa and OTUB1 protein levels in HCT116 and $\mathrm{Caco} 2$ cells were measured by western blot (B, D, F). Data were normalized to $\beta$-actin. The data represent the mean of three independent experiments, and the error bars represent the SD. *, $\mathrm{P}<0.05 ; * *, \mathrm{P}<0.01 ; * * *, \mathrm{P}<0.001$.

A

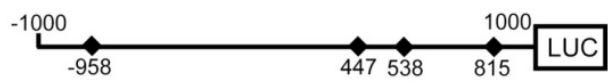

B

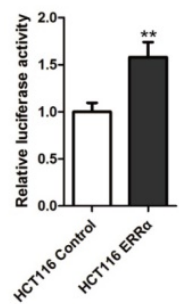

$\mathrm{E}$

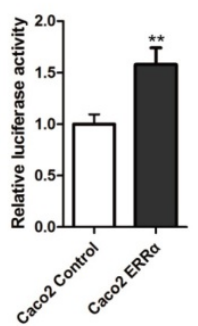

C

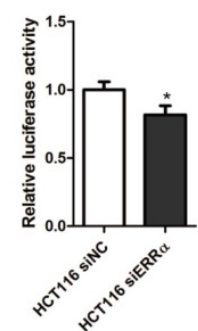

$\mathrm{F}$

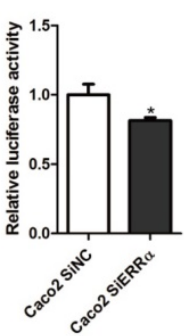

D

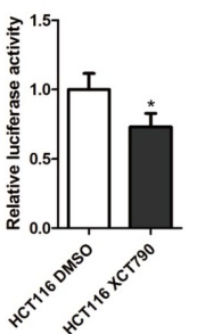

G

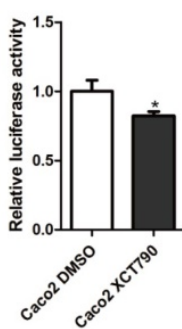

Figure 2. ERR $\alpha$ induces OTUBI promoter activity. (A) Scheme representing the cloning of OTUBI promoter sequence $(-1000$ to +1000$)$ into the PGL3 reporter. PGL3 or PGL3-OTUBI were co-transfected with empty vector or an ERRa expression plasmid, DMSO or XCT790 $(10 \mu \mathrm{M})$, siNC or ERRa siRNA for $48 \mathrm{~h}$. The luciferase activity in HCT116 (B, C, D) or Caco2 (E, F, G) cells was then measured. Data were normalized to Renilla activity. The data represent the mean of three independent experiments, and the error bars represent the SD. *, P $<0.05$; **, $P<$ 0.01 .

Four potential ERRa response elements (ERREs) were found within OTUB1 promoter: ERRE-S1 (TGGCCTTGA), located at -958/-950 bp from the transcription initiation site; ERRE-S2 (TGACCTTTA) located at 447/455 bp; ERRE-S3 (TCAAGATGA)

located at 538/546 bp; and ERRE-S4 (TGACCTTGA) located at $815 / 823 \mathrm{bp}$ (Table S2). To determine which of these was bound by ERRa, a series of truncated OTUB1 promoter constructs were generated and co-transfected with the ERRa expression vector or empty vector. We found that the activity of the constructs with the $-1000 /+1000,+339 /+1000$, $+485 /+1000,+658 /+1000$ OTUB1 promoter sequences were similar, indicating that they all contained the ERRa binding site. However, the activity of the reporter with the $+900 /+1000$ OTUB1 promoter sequence was not increased by ERRa (Figure 3A). We also mutated the putative ERRa binding sites and, again, conducted luciferase reporter assays after their co-transfection with the ERRa expression vector or empty vector (Figure 3B). We found that the mutation of ERRE-S1, ERRE-S2 or ERRE-S3 did not affect the luciferase activity, while the mutation of ERRE-S4 caused a significant decrease in the luciferase activity, suggesting that ERRa binding site was indeed ERRE-S4.

To further demonstrate that ERRa binds to the OTUB1 promoter through ERRE-S4, we conducted ChIP assays in HCT116 cells and used primers amplifying the $+670 /+901$ region of OTUB1 promoter, containing ERRE-S4 (Figure 4A). As shown in Figure 4B, ChIP assays showed a significant enrichment of the fragment that contained the ERRE-S4 site in the samples incubated with the anti-ERRa only. These results confirmed the interaction between ERRa and the ERRES4 site on OTUB1 promoter. 


\section{ERRa promotes CRC cell migration in an OTUB 1-dependent manner}

To assess the function of ERRa and OTUB1 in CRC cell migration, transwell assays were performed. First, the effect of siRNAs of OTUB1 was tested by western blot (Figure S3). As show in Figure 5A and C, the migration ability of ERRa-overexpressing cells was enhanced, and the knockdown of OTUB1 reversed this effect. In contrast, silencing of ERRa significantly suppressed the migration of HCT116 and Caco2 cells, and this effect was reversed by overexpression of OTUB1 (Figure 5B and D).

A
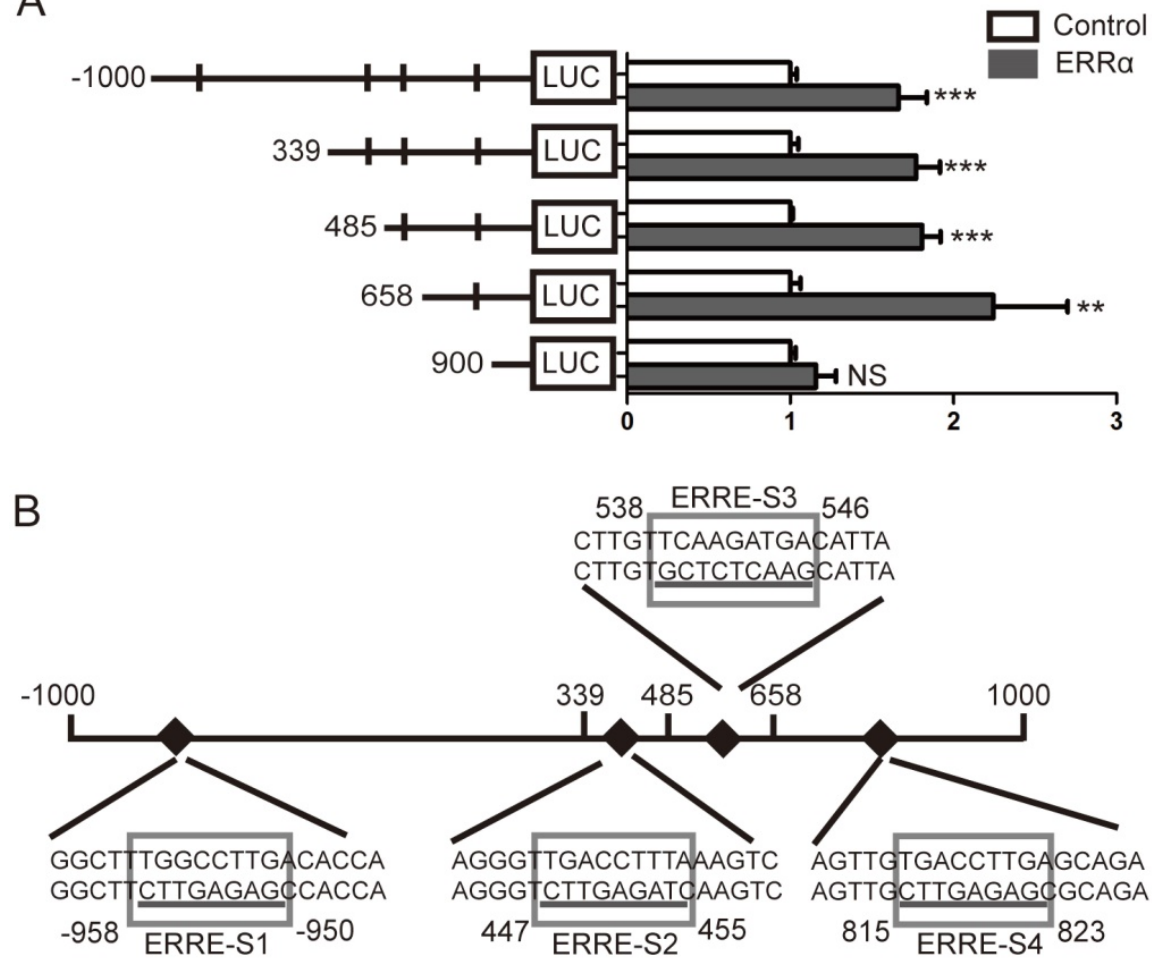

C

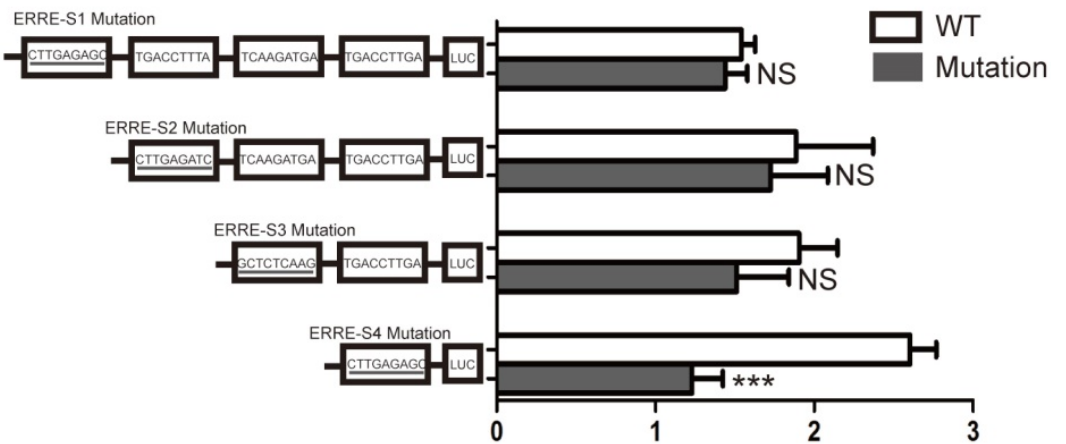

Figure 3. Identification of putative ERR $\alpha$ binding sites on OTUBI promoter. (A) An empty vector or an ERRa expression plasmid was co-transfected with constructs containing different lengths of OTUBI promoter $(-1000 /+1000,+339 /+1000,+485 /+1000,+658 /+1000,+900 /+1000)$ cloned in a luciferase reporter plasmid into HCT116 cells for $48 \mathrm{~h}$. The cells were then lysed and assayed for luciferase activity. Data were normalized to Renilla activity. (B) Diagram of the OTUBI locus showing ERRE-S1 4 in the promoter and the mutation sequences. (C) An empty vector or ERR $\alpha$ expression plasmid was co-transfected with wild-type or mutated OTUB I-luciferase constructs into HCT11 6 cells for $48 \mathrm{~h}$. The luciferase activity was then assayed. Data were normalized to Renilla activity. The data represent the mean of three independent experiments, and the error bars represent the SD. **, $\mathrm{P}<0.01$; ***, $\mathrm{P}<$ 0.001 .

\section{ERRa promotes CRC cell migration through}

Finally, we investigated how the effect of ERRa on OTUB1 promotes CRC cells migration, which is hand, knockdown of ERRa expression significantly decreased vimentin levels and, again, this effect was reversed by OTUB1 overexpression (Figure 6B and D). However, we did not detect changes in the expression of E-cadherin, $\beta$-catenin, snail, and zo-1 (Figure S4). These results showed that ERRa regulates expression of OTUB1 and promotes colorectal cell migration through vimentin.

\section{Discussion}

In this study, we demonstrated that ERRa regulates OTUB1 expression by binding the OTUB1 promoter and promotes migration of CRC cells through vimentin. Recent studies have reported the oncogenic role OTUB1 in many cancers, such as prostate, ovarian, breast and lung cancer, esophageal squamous cell carcinoma (ESCC), hepatocellular carcinoma (HCC), and CRC. OTUB1 mediates prostate cancer tumorigenesis and invasion through RhoA activation [24]. Additionally, it promotes tumor progression and chemotherapy resistance by inhibiting FOXM1 degradation in ovarian cancer and breast cancer [25, 26]. Moreover, in lung cancer, by inhibiting RAS ubiquitination, OTUB1 triggers cancer development [27]. OTUB1 expression is also associated with a malignant phenotype in digestive tract cancer. In ESCC, OTUB1 predicts poor prognosis and facilitates metastasis stabilizing Snail and promoting EMT [28]. OTUB1 is highly expressed in HCC, and promotes HCC cells proliferation and invasion [29]. In gastric cancer, OTUB1 is an independent risk factor for disease-specific survival 
and enhances tumor invasiveness [30]. In CRC, OTUB1 induces metastasis by regulating EMT [22]. OTUB1 is overexpressed in prostate cancer, ovarian cancer, breast cancer, ESCC, HCC, gastric and CRC. However, the molecular mechanism underlying OTUB1 overexpression has not been clarified. Yuan and collogues have shown that the microRNA miR-542-3p targets OTUB1 and inhibits cell proliferation and invasion in CRC [31]. In this study, we found that ERRa regulates OTUB1 expression in CRC by binding to OTUB1 promoter.

ERRa has been shown to be important for cell metabolism, tumorigenesis, cancer proliferation and metastasis. ERRa is overexpressed in CRC tissues and high ERRa levels indicate poor prognosis $[15,16]$. By analyzing the TGCA database, we found that the expression of OTUB1 is highly related to that of $E R R a$. ERRa is an orphan nuclear receptor which binds to TNAAGGTCA sequences on DNA. By binding to their promoters, ERRa can regulate many genes in colorectal cell lines, such as Osteopontin (OPN) in HT29 cells [32] and SULT2A1 in Caco-2 cells [33]. Here, we found four putative ERREs on OTUB1 promoter. Overexpression or downregulation of
ERRa in HCT116 and Caco2 cells increased or decreased promoter activity, and regulated OTUB1 expression. Deletion and mutation analyses of OTUB1 promoter and CHIP assays revealed that ERRa regulates OTUB1 expression by binding to ERREE-S4 on OTUB1. Therefore, OTUB1 is a novel target of ERRa in CRC.

A

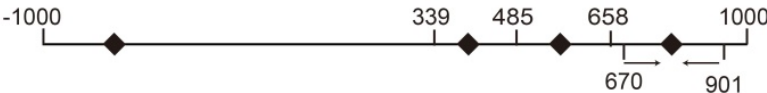

B

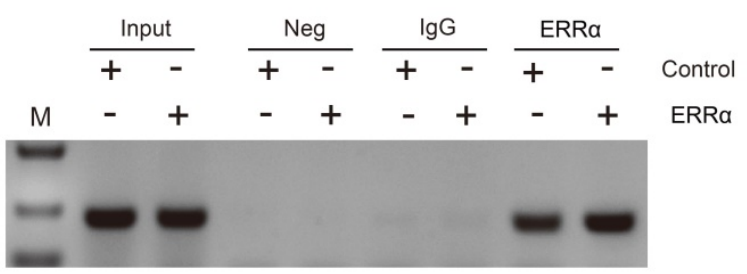

Figure 4. ERRa binds to ERRE-S4 on OTUBI promoter. (A) Schematic representation of the possible PCR products obtained after CHIP. (B) HCT116 cells were transfected with an empty vector or an ERR $\alpha$ expression plasmid for $48 \mathrm{~h}$, and ChIP assays were performed using a human ERRa-specific antibody and the purified DNA was amplified by PCR. Input or negative control (NC) were not treated with antibody or were incubated with rabbit IgG, respectively.
A

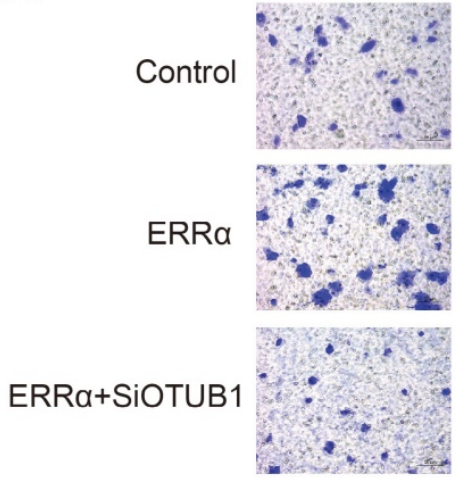

C

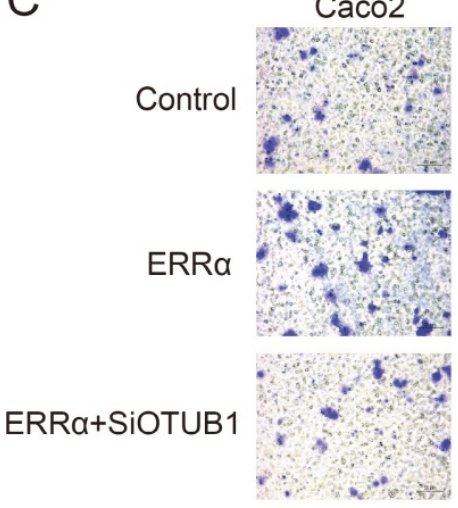

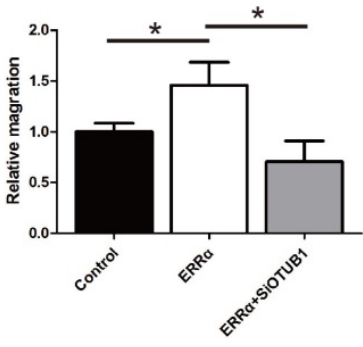

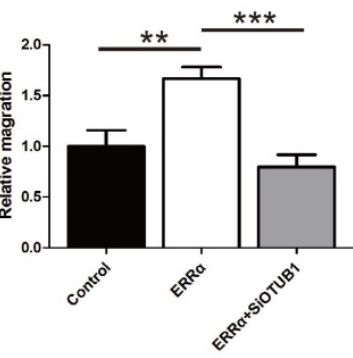

B

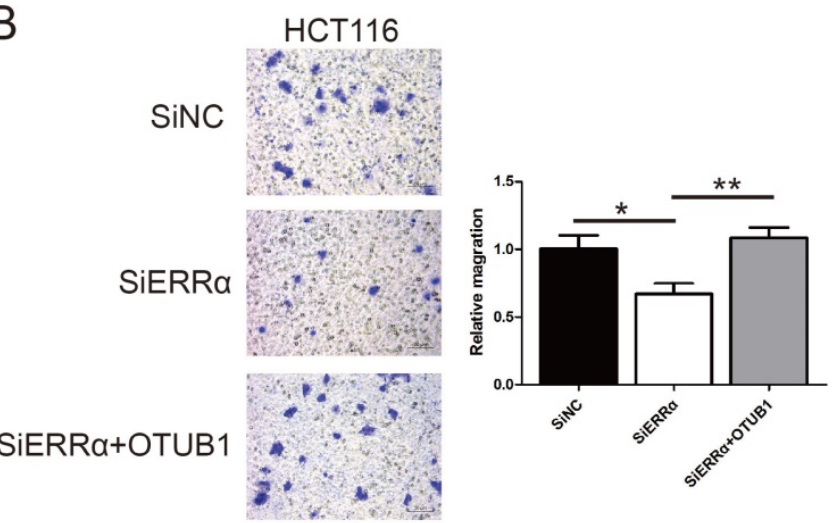

D

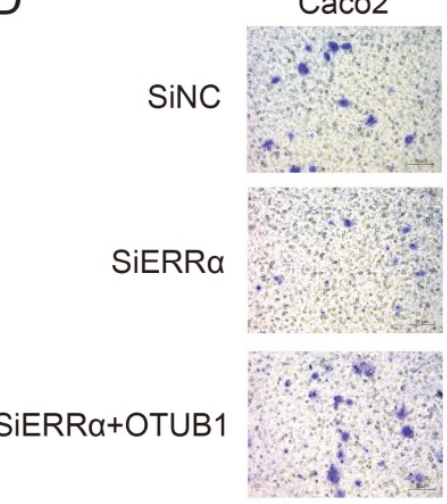

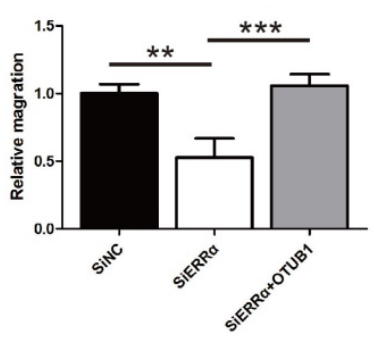

Figure 5. ERRa promotes CRC cell migration via OTUB 1. HCT116 (A) or Caco2 (C) cells were transfected with ERRa expression plasmid (or empty vector) with or without OTUB1 siRNAs for $24 \mathrm{~h}$. Then, $0.5 \times 10^{5} \mathrm{HCT} 116$ cells or $1.5 \times 10^{5} \mathrm{Caco} 2$ were seeded into a Boyden chamber for 24 to $36 \mathrm{~h}$ to assay cell migration. HCT116 (B) or Caco2 (D) cells were transfected with an ERRa siRNA (or siNC) with or without OTUB1 expression plasmid for $24 \mathrm{~h}$. Then, $0.5 \times 10^{5} \mathrm{HCT} 116$ cells or $1.5 \times 10^{5} \mathrm{Caco} 2 \mathrm{were}$ seeded into a Boyden chamber for 24 to $36 \mathrm{~h}$ to assay cell migration. Representative images are shown. The relative magration of cells is indicated on the right. Bars represent the mean \pm SD of three independent experiments. *, $\mathrm{P}<0.05 ; * *, \mathrm{P}<0.01 ; * * *, \mathrm{P}<0.001$. 
A

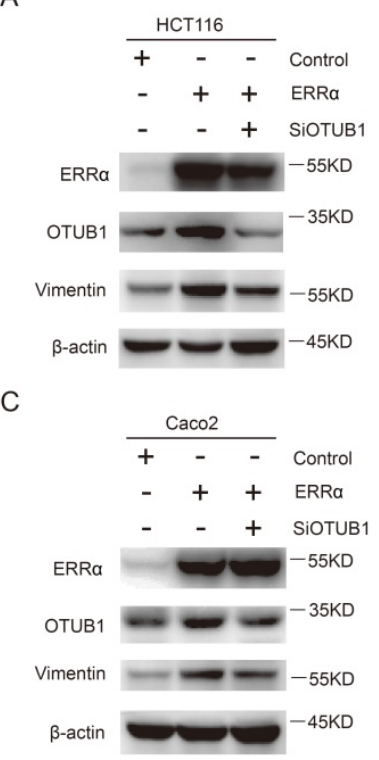

B

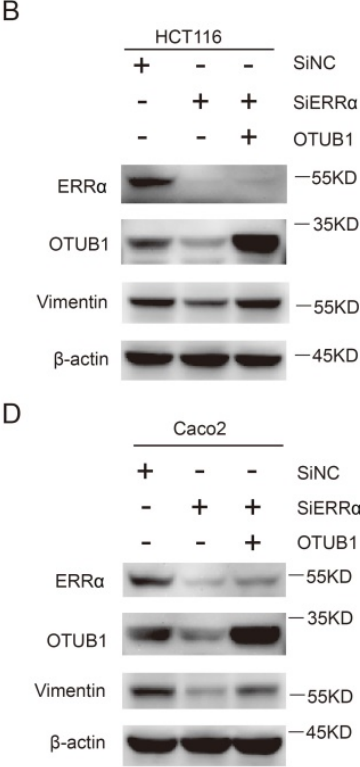

Figure 6. ERRa promotes CRC cell migration through vimentin. HCT116 (A) or Caco2 (C) cells were transfected with ERRa expression plasmid (or empty vector) with or without OTUB1 siRNAs for $48 \mathrm{~h}$. The protein levels of ERRa, OTUB1 and vimentin were measured by western blot. HCT116 (B) or Caco2 (D) cells were transfected with an ERRa siRNA (or siNC) with or without OTUB1 expression plasmid for $48 \mathrm{~h}$. The protein levels of ERRa, OTUBI, and vimentin were measured by western blot.

Previous studies have shown that ERRa promotes CRC cell proliferation by targeting a large set of key genes related to glycolysis, tricarboxylic acid cycle and lipid synthesis [34]. Moreover, ERRa promotes the proliferation and migration of colorectal cells by upregulating IL- 8 and thus inducing the phosphorylation of ERK1/2 and STAT3 [35]. In lung cancer, ERRa induces EMT by regulating Slug and promotes the migration and invasion of A549 cells [36]. In triple-negative breast cancer, ERRa can directly bind the fibronectin promoter, not Snail or Slug promoters, and promote EMT [37]. In this study, we demonstrated that ERRa targets OTUB1 and promotes cells migration through vimentin. Vimentin is an intermediate filament protein that is expressed in mesenchymal cells and a marker for EMT. Vimentin overexpression in epithelial cells indicates enhanced migration ability. In CRC, high vimentin expression predicts poor disease-free survival and overall survival [38] and it is a biomarker for lymph node metastasis [39]. In SW480 and SW620 cells models, OTUB1 can regulate EMT molecular markers such as E-cadherin and vimentin [22]. However, in this model (HCT116 and Caco2 cells), ERRa upregulated only vimentin via OTUB1, but not other EMT markers.

\section{Conclusions}

Our findings demonstrate that ERRa promotes the migration of CRC cells through OTUB1. By

binding OTUB1 promoter, ERRa regulates OTUB1 expression and promotes EMT of colorectal cells. Therefore, targeting ERRa and OTUB1 might be a new strategy for CRC treatment.

\section{Abbreviations}

ERRa: estrogen-related receptor alpha; OTUB1: ovarian tumor domain-containing ubiquitin aldehyde binding protein 1; CRC: colorectal cancer; ESCC: esophageal squamous cell carcinoma; HCC: hepatocellular carcinoma; ERREs: ERRa response elements; CHIP: Chromatin Immunoprecipitation.

\section{Supplementary Material}

Supplementary figures and tables. http://www.jcancer.org/v10p5812s1.pdf

\section{Acknowledgments}

This work was supported by the National Natural Science Foundation of China [grant number 81502045].

\section{Competing Interests}

The authors have declared that no competing interest exists.

\section{References}

1. Chen W, Zheng R, Baade PD, et al. Cancer statistics in China, 2015. CA Cancer J Clin. 2016; 66: 115-32.

2. Giguere V. Orphan nuclear receptors: from gene to function. Endocr Rev. 1999; 20: 689-725.

3. Deblois G, Hall JA, Perry MC, et al. Genome-wide identification of direct target genes implicates estrogen-related receptor alpha as a determinant of breast cancer heterogeneity. Cancer Res. 2009; 69: 6149-57.

4. Cai $\mathrm{O}$, Lin $\mathrm{T}$, Kamarajugadda $\mathrm{S}$, et al. Regulation of glycolysis and the Warburg effect by estrogen-related receptors. Oncogene. 2013; 32: 2079-86.

5. Giguere V. Transcriptional control of energy homeostasis by the estrogen-related receptors. Endocr Rev. 2008; 29: 677-96.

6. Luo J, Sladek R, Carrier J, et al. Reduced fat mass in mice lacking orphan nuclear receptor estrogen-related receptor alpha. Mol Cell Biol. 2003; 23: 7947-56.

7. Tennessen JM, Baker KD, Lam G, et al. The Drosophila estrogen-related receptor directs a metabolic switch that supports developmental growth. Cell Metab. 2011; 13: 139-48.

8. Sailland J, Tribollet V, Forcet C, et al. Estrogen-related receptor alpha decreases RHOA stability to induce orientated cell migration. Proc Natl Acad Sci U S A. 2014; 111: 15108-13.

9. Dwyer MA, Joseph JD, Wade HE, et al. WNT11 expression is induced by estrogen-related receptor alpha and beta-catenin and acts in an autocrine manner to increase cancer cell migration. Cancer Res. 2010; 70: 9298-308.

10. Jarzabek K, Koda M, Kozlowski L, et al. The significance of the expression of ERRalpha as a potential biomarker in breast cancer. J Steroid Biochem Mol Biol. 2009; 113: 127-33.

11. Sun P, Sehouli J, Denkert C, et al. Expression of estrogen receptor-related receptors, a subfamily of orphan nuclear receptors, as new tumor biomarkers in ovarian cancer cells. J Mol Med (Berl). 2005; 83: 457-67.

12. Mori T, Sawada M, Kuroboshi $\mathrm{H}$, et al. Estrogen-related receptor alpha expression and function are associated with vascular endothelial growth factor in human cervical cancer. Int J Gynecol Cancer. 2011; 21: 609-15.

13. Fujimura $\mathrm{T}$, Takahashi $\mathrm{S}$, Urano $\mathrm{T}$, et al. Increased expression of estrogen-related receptor alpha (ERRalpha) is a negative prognostic predictor in human prostate cancer. Int J Cancer. 2007; 120: 2325-30.

14. Tiwari A, Shivananda S, Gopinath KS, et al. MicroRNA-125a reduces proliferation and invasion of oral squamous cell carcinoma cells by targeting estrogen-related receptor alpha: implications for cancer therapeutics. J Biol Chem. 2014; 289: 32276-90.

15. Cavallini A, Notarnicola M, Giannini R, et al. Oestrogen receptor-related receptor alpha (ERRalpha) and oestrogen receptors (ERalpha and ERbeta) exhibit different gene expression in human colorectal tumour progression. Eur J Cancer. 2005; 41: 1487-94. 
16. Liang R, Lin $\mathrm{Y}$, Yuan CL, et al. High expression of estrogen-related receptor alpha is significantly associated with poor prognosis in patients with colorectal cancer. Oncol Lett. 2018; 15: 5933-9.

17. Lin JT, Lineberry NB, Kattah MG, et al. Naive CD4 t cell proliferation is controlled by mammalian target of rapamycin regulation of GRAIL expression. J Immunol. 2009; 182: 5919-28.

18. Sun $X X$, Challagundla KB, Dai MS. Positive regulation of $p 53$ stability and activity by the deubiquitinating enzyme Otubain 1. EMBO J. 2012; 31: 576-92.

19. Chen Y, Wang YG, Li Y, et al. Otub1 stabilizes MDMX and promotes its proapoptotic function at the mitochondria. Oncotarget. 2017; 8: 11053-62.

20. Herhaus L, Al-Salihi M, Macartney T, et al. OTUB1 enhances TGFbeta signalling by inhibiting the ubiquitylation and degradation of active SMAD2/3. Nat Commun. 2013; 4: 2519.

21. Goncharov T, Niessen $\mathrm{K}$, de Almagro MC, et al. OTUB1 modulates c-IAP1 stability to regulate signalling pathways. EMBO J. 2013; 32: 1103-14.

22. Zhou Y, Wu J, Fu X, et al. OTUB1 promotes metastasis and serves as a marker of poor prognosis in colorectal cancer. Mol Cancer. 2014; 13: 258

23. Wang $\mathrm{H}, \mathrm{Wu} \mathrm{J}$, Meng X, et al. MicroRNA-342 inhibits colorectal cancer cell proliferation and invasion by directly targeting DNA methyltransferase 1 . Carcinogenesis. 2011; 32: 1033-42.

24. Iglesias-Gato D, Chuan YC, Jiang N, et al. OTUB1 de-ubiquitinating enzyme promotes prostate cancer cell invasion in vitro and tumorigenesis in vivo. Mol Cancer. 2015; 14: 8

25. Wang Y, Zhou X, Xu M, et al. OTUB1-catalyzed deubiquitination of FOXM1 facilitates tumor progression and predicts a poor prognosis in ovarian cancer. Oncotarget. 2016; 7: 36681-97.

26. Karunarathna U, Kongsema M, Zona S, et al. OTUB1 inhibits the ubiquitination and degradation of FOXM1 in breast cancer and epirubicin resistance. Oncogene. 2016; 35: 1433-44.

27. Baietti MF, Simicek M, Abbasi Asbagh L, et al. OTUB1 triggers lung cancer development by inhibiting RAS monoubiquitination. EMBO Mol Med. 2016; 8: 288-303.

28. Zhou H, Liu Y, Zhu R, et al. OTUB1 promotes esophageal squamous cell carcinoma metastasis through modulating Snail stability. Oncogene. 2018; 37: 3356-3368.

29. Ni Q, Chen J, Li X, et al. Expression of OTUB1 in hepatocellular carcinoma and its effects on HCC cell migration and invasion. Acta Biochim Biophys Sin (Shanghai). 2017; 49: 680-688.

30. Weng $\mathrm{W}$, Zhang $\mathrm{Q}, \mathrm{Xu} \mathrm{M}$, et al. OTUB1 promotes tumor invasion and predicts a poor prognosis in gastric adenocarcinoma. Am J Transl Res. 2016; 8: 2234-44.

31. Yuan $L$, Yuan $P$, Yuan $H$, et al. miR-542-3p inhibits colorectal cancer cell proliferation, migration and invasion by targeting OTUB1. Am J Cancer Res. 2017; 7 : 159-172.

32. Boudjadi S, Bernatchez G, Beaulieu JF, et al. Control of the human osteopontin promoter by ERRalpha in colorectal cancer. Am J Pathol. 2013; 183: 266-276.

33. Huang C, Zhou T, Chen Y, et al. Estrogen-related receptor ERRalpha regulation of human hydroxysteroid sulfotransferase (SULT2A1) gene expression in human Caco-2 cells. J Biochem Mol Toxicol. 2014; 28: 32-8.

34. Simpson LO, Shand BO. Glomerular permeability. Clin Sci. 1986; 71: 221-223.

35. Ding S, Tang Z, Jiang Y, et al. IL-8 Is Involved in Estrogen-Related Receptor alpha-Regulated Proliferation and Migration of Colorectal Cancer Cells. Dig Dis Sci. 2017; 62: 3438-3446.

36. Huang JW, Guan BZ, Yin LH, et al. Effects of estrogen-related receptor alpha (ERRalpha) on proliferation and metastasis of human lung cancer A549 cells. J Huazhong Univ Sci Technolog Med Sci. 2014; 34: 875-881.

37. Wu YM, Chen ZJ, Liu H, et al. Inhibition of ERRalpha suppresses epithelial mesenchymal transition of triple negative breast cancer cells by directly targeting fibronectin. Oncotarget. 2015; 6: 25588-601.

38. Du L, Li J, Lei L, et al. High Vimentin Expression Predicts a Poor Prognosis and Progression in Colorectal Cancer: A Study with Meta-Analysis and TCGA Database. Biomed Res Int. 2018; 6387810.

39. Toiyama $\mathrm{Y}$, Yasuda $\mathrm{H}$, Saigusa $\mathrm{S}$, et al. Increased expression of Slug and Vimentin as novel predictive biomarkers for lymph node metastasis and poor prognosis in colorectal cancer. Carcinogenesis. 2013; 34: 2548-2557. 